\title{
Paternalisme et territoires politiques dans la France de la seconde révolution industrielle : Un regard rétrospectif sur les liens entre firmes et territoires communaux
}

Industrial paternalism and political territories in France during second industrial revolution: A retrospective about links between firms and municipalities

Paternalismus und politische Territorien in Frankreich des zweiten Industriezeitalters : Eine rückblickende Betrachtung über die Verbindungen zwischen den Firmen und den Gemeindegebieten

\section{Simon Edelblutte}

\section{OpenEdition \\ Journals}

Édition électronique

URL : http://journals.openedition.org/rge/3043

DOI : $10.4000 /$ rge.3043

ISSN : 2108-6478

Éditeur

Association des géographes de l'Est

Édition imprimée

Date de publication : 31 décembre 2010

ISSN : 0035-3213

\section{Référence électronique}

Simon Edelblutte, «Paternalisme et territoires politiques dans la France de la seconde révolution industrielle : Un regard rétrospectif sur les liens entre firmes et territoires communaux », Revue Géographique de l'Est [En ligne], vol. 50 / 3-4 | 2010, mis en ligne le 17 octobre 2011, consulté le 08 septembre 2020. URL : http://journals.openedition.org/rge/3043 ; DOI : https://doi.org/10.4000/rge. 3043

Ce document a été généré automatiquement le 8 septembre 2020

Tous droits réservés 


\title{
Paternalisme et territoires
} politiques dans la France de la seconde révolution industrielle : Un regard rétrospectif sur les liens entre firmes et territoires

\section{communaux}

\author{
Industrial paternalism and political territories in France during second \\ industrial revolution: A retrospective about links between firms and \\ municipalities \\ Paternalismus und politische Territorien in Frankreich des zweiten \\ Industriezeitalters : Eine rückblickende Betrachtung über die Verbindungen \\ zwischen den Firmen und den Gemeindegebieten
}

Simon Edelblutte

\section{Introduction}

1 Lors du décès d'Yves Rocher, fondateur de l'entreprise de produits de beauté du même nom, en décembre 2009, l'hebdomadaire Le Point titrait à propos de la ville d'implantation de l'entreprise : "Mort d'Yves Rocher : La Gacilly, fief breton du géant de la beauté" (www.lepoint.fr, 2009).

2 En utilisant dans son titre le mot «fief " pour caractériser la commune de La Gacilly (Bretagne), le journaliste rend compte d'un lien extrêmement fort entre une firme et sa commune d'attache, territoire politique de la grande échelle. Si ces liens existent encore aujourd'hui, comme en témoigne cet exemple breton, ils étaient encore 
beaucoup plus forts au temps du paternalisme des XIX ${ }^{\text {ème }}$ et XX ${ }^{\text {ème }}$ siècles et c'est l'objet de ce travail.

3 Si de nombreux articles et ouvrages traitent les liens entre entreprises et territoires locaux à diverses échelles (Vandermotten, 2004 ; Houssel, 1992; Maisonniac, 2000 ; Donze, 2001), notamment sur un plan économique, social ou spatial, plus rares sont les travaux liant la firme au territoire politique de la grande échelle, c'est-à-dire à la commune en France. En effet, la taille réduite $\left(14,88 \mathrm{~km}^{2}\right.$ de moyenne), et surtout la très faible population de nombre d'entre-elles (certaines sont même inhabitées), en font des territoires politiques très souvent critiqués, réputés archaïques (Dréano, 1997) et souvent dénoncés comme figés depuis la Révolution. "Il n'y a plus guère de fondement économique à cet univers des communes françaises " (Di Méo, 1993) et l'on constate donc une "inadéquation entre le territoire civique légal où intervient la municipalité et le territoire du quotidien " (Dréano, 1996-98).

4 Ces liens entre territoire politique de la grande échelle et entreprise sont, historiquement, un des éléments du paternalisme qui s'est largement développé dans la France des XIX ${ }^{\text {ème }}$ et $\mathrm{XX}^{\text {ème }}$ siècle. Le paternalisme se définit le plus souvent comme une "attitude protectrice du patron envers l'ouvrier, comparable à celle d'un père de famille envers ses enfants "(Brand, Durousset, 1991).Plus généralement, cela revient à se conduire comme un père envers d'autres personnes sur lesquelles on exerce ou tente d'exercer une autorité. Sa définition et son sens ont aujourd'hui une connotation péjorative prononcée qui n'existait pas à l'époque, l'essentiel des ouvriers étant largement consentant face à cette politique. Elle est en effet une amélioration sensible de leur sort par rapport à ce qui prévalait au temps proto-industriels ou surtout lors de la première révolution industrielle, où la dimension sociale des entreprises est encore loin d'être aboutie. Le pas de temps de 1850/1950, regroupant approximativement seconde révolution industrielle et fordisme, correspond donc l'apogée du paternalisme.

5 Cette implication très forte de l'industriel ${ }^{1}$ dans la vie des ses employés, à une époque où entreprise et dirigeant se confondent souvent, a une traduction territoriale qui sera au cœur de ce travail. Il s'agit donc ici de comprendre en quoi ces firmes du passé ontelles façonné les territoires de très nombreuses communes?

Pour ce type de travail, les sources bibliographiques historiques sont essentielles, notamment les ouvrages locaux, mais aussi les listes de maires, de députés, de conseillers généraux, fournies par certains ouvrages ou encore par certains sites internet, notamment généalogiques (www.francegenweb.org). Cependant, ces sites sont encore incomplets et aucun ne recense encore tous les maires depuis $1789 . .$.

7 Pour les modifications territoriales des communes, le recours aux fichiers de l'Institut National de la Statistique et des Études Économiques (INSEE) qui récole toutes les modifications de territoires administratifs depuis la fin de la seconde guerre mondiale, est indispensable. Pour les périodes plus anciennes, la collection «Paroisses et communes de France» initié en 1974 (Dupaquier et al.) recense, par département, toutes les disparitions, créations, fusions, dé-fusions de communes depuis 1789.

8 Cependant, cette collection est incomplète et ne concerne que 41 départements sur les 96. Elle semble de plus stoppée depuis 2000. Ainsi, pour certaines modifications, c'est essentiellement soit l'utilisation des recensements (une commune qui n'est pas recensée avant telle ou telle date n'existait évidemment pas avant), soit l'observation des cartes topographiques du Service Géographique des Armées (SGA) et de l'Institut 
Géographique National (IGN) qui permettent de repérer les modifications, notamment mineures (changement de limite communale par exemple) des territoires communaux.

Dans cette étude, les exemples lorrains ont été privilégiés, car ils sont évidemment nombreux dans une région de forte tradition industrielle. Il en existe cependant beaucoup d'autres partout ailleurs en France et certains seront d'ailleurs évoqués.

Pour débuter ce travail, il faut dans un premier temps comprendre de quelle façon et pourquoi les industriels du XIX ${ }^{\text {ème }}$ et XX ${ }^{\text {ème }}$ siècles se sont impliqués dans la politique locale, puis, dans un second temps, comment ils en sont venus à transformer leurs communes d'accueil, par des aménagements concrets, construisant des villes entières. Enfin, répondant à la question posée plus haut, la troisième partie s'attachera à montrer comment les firmes de l'époque ont modifié, voire parfois créé, de nouveaux territoires politiques de la grande échelle, adaptant la trame communale au fonctionnement des territoires, alors qu'à petite échelle, c'est surtout l'inverse qui se produit.

\section{I - Les industriels s'investissent dans la politique}

\section{A - Pourquoi un tel investissement?}

Figure 1 : Parlementaires-industriels dans les Vosges de la $3^{\text {ème }}$ République

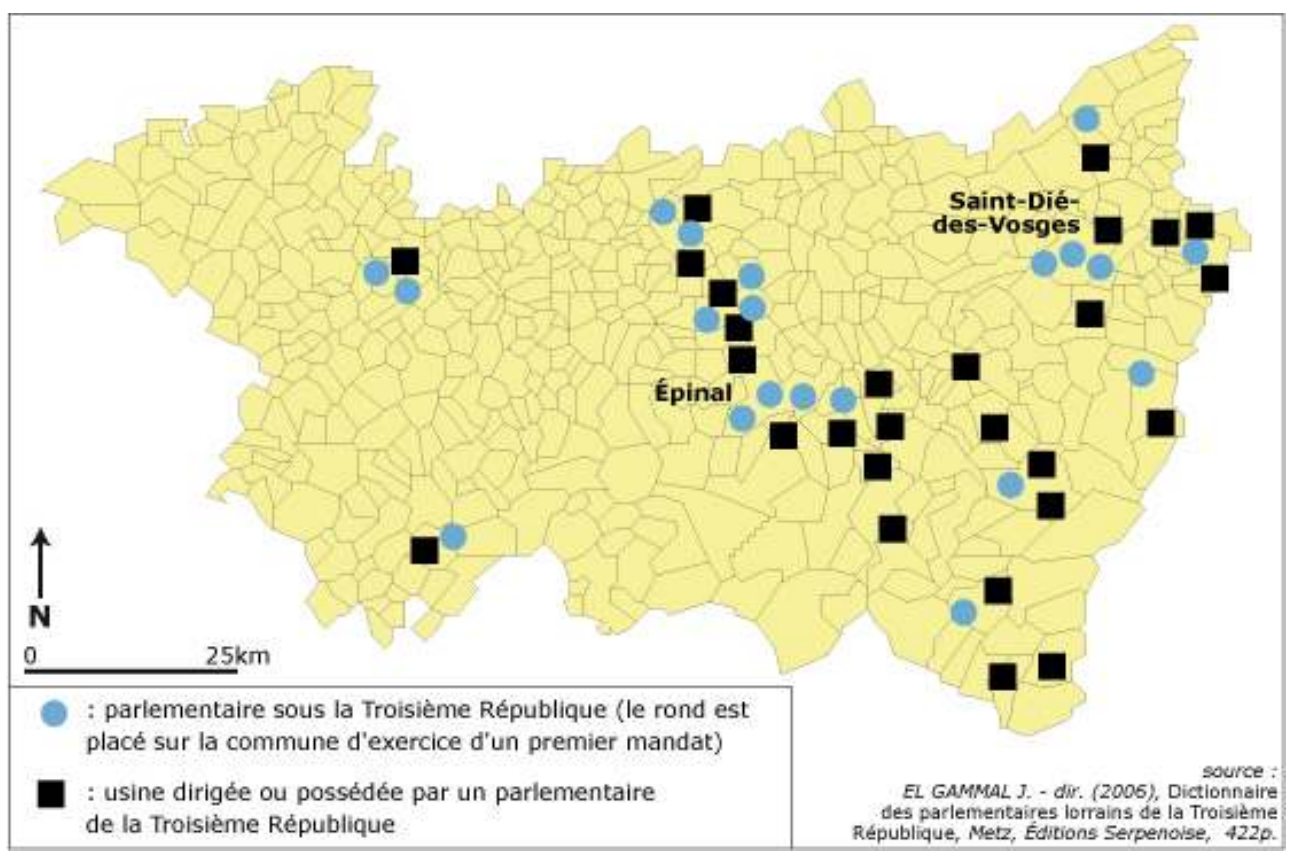

11 La liste des industriels du XIX ${ }^{\text {ème }}$ siècle s'investissant dans la politique locale est impressionnante. Rien que dans le département des Vosges, pris ici en exemple (fig. 1), et durant la Troisième République, au moins 20 députés ou sénateurs, sur un liste de 58 parlementaires (soit 35\%) sont aussi ou ont été des industriels (El Gammal - dir., 2006), principalement dans la partie la plus industrielle du département.

Ces fonctions électives élevées sont occupées par les industriels qui peuvent ainsi agir à un niveau national, avec un impact souvent plus diffus sur les territoires locaux. C'est donc plutôt la fonction de maire ou celle de conseiller général qui permet une action 
locale forte. Cependant, à ces échelons inférieurs (départemental, communal), il devient impossible de recenser et cartographier précisément, faute d'informations exhaustives (liste de maires de chaque commune depuis la Révolution), le nombre d'industriels conseillers généraux ou, surtout, maires.

Certains exemples précis, mêlant fonctions locales, départementales et nationales, peuvent être néanmoins développés et sont particulièrement révélateurs, comme celui présenté sur le tableau 1 liant les politiciens-industriels par les femmes. Il n'était pas rare en effet que les familles patronales s'allient entre-elles par le mariage, préservant ou agrandissant ainsi le capital familial (Poull, 1982 ; Durupt, 1990).

Tableau 1 : Un exemple de dynastie d'hommes politiques-industriels vosgiens

\begin{tabular}{|c|c|c|}
\hline Nom & Activité industrielle & Activité politique \\
\hline $\begin{array}{l}\text { Christian KIENER } \\
(1807-1896)\end{array}$ & $\begin{array}{l}\text { - filature à Monthureux-sur- } \\
\text { Saône } \\
\text { - tissage et filature à Eloyes }\end{array}$ & $\begin{array}{l}\text { - conseiller municipal à } \\
\text { Monthureux-sur-Saône } \\
\text { - conseiller général du canton de } \\
\text { Monthureux-sur-Saône } \\
\text { - maire d'Éloyes } \\
\text { - maire d'Épinal } \\
\text { - sénateur des Vosges }\end{array}$ \\
\hline $\begin{array}{c}\text { Edouard BRESSON } \\
(1826-1911) \\
\text { beau-père }\end{array}$ & $\begin{array}{l}\text { - filature à Monthureux-sur- } \\
\text { Saône }\end{array}$ & $\begin{array}{l}\text { - maire de Monthureux-sur-Saône } \\
\text { - conseiller général du canton de } \\
\text { Darney } \\
\text { - député des Vosges }\end{array}$ \\
\hline $\begin{array}{l}\text { Léon GAUTIER } \\
(1848-1933)\end{array}$ & $\begin{array}{l}\text { - filature à Golbey } \\
\text { - tissage à Nomexy }\end{array}$ & $\begin{array}{l}\text { - conseiller municipal de Claudon } \\
\text { - conseiller général du canton de } \\
\text { Monthureux-sur-Saône } \\
\text { - député des Vosges }\end{array}$ \\
\hline
\end{tabular}

14 C'est justement sur les territoires communaux que l'influence des industriels est la plus sensible. Le poste de maire, électif depuis 1871 dans les communes de moins de 20000 habitants et non chefs-lieux (canton ou département) et depuis 1882 pour toutes les communes, est en effet le premier à être exercé par les industriels. Les causes de cet intérêt manifesté pour cette fonction par les industriels sont externes et internes à l'entreprise ; elles se mêlent largement et il est difficile de les hiérarchiser, d'autant plus qu'elles sont sujettes à des interprétations diverses en fonction des opinions politiques des auteurs :

- les causes externes sont les besoins politiques de communes aux habitants mal armés pour faire face à une mutation rapide de leur population et de leur fonctionnement. Ainsi, le personnel politique des communes rurales est constitué essentiellement de paysans qui, s'ils étaient parfaitement capables de gérer les communes agricoles d'avant l'industrialisation, ne réussissent pas à s'adapter à la gestion de communes brusquement grossies par l'apport d'ouvriers aux préoccupations forts différentes de leurs administrés habituels. C'est donc souvent par manque de personnel politique - et donc par pragmatisme - que les communes concernées par l'industrialisation se tournent vers l'industriel pour l'élire au poste de maire. À cela s'ajoutent des motivations plus complexes et subtiles, liées à la puissance locale qu'exerce l'industriel et à son image de notable ;

- les causes internes à l'entreprise sont les plus évidentes et les plus soulignées alors que les précédentes ont joué un rôle tout aussi important. Les industriels ont en effet un intérêt économique et social certain à devenir maires des communes d'accueil de leurs usines : le contrôle de la mairie permet d'orienter les investissements vers des aménagements 
profitables à l'entreprise, permet de mieux contrôler la population hors du temps de travail par l'action de maintien de l'ordre, etc.

Néanmoins, ce contrôle, souvent présenté aujourd'hui comme une aliénation de la liberté des ouvriers par rapport à un industriel quelque peu omnipotent, doit être nuancé. En effet, les industriels les plus éclairés de l'époque, inspirés par le mouvement du catholicisme social, ont une haute idée de leur rôle vis-à-vis d'ouvrier qu'ils veulent avant tout protéger et non pas forcément contrôler. De plus, les industriels ont aussi une volonté de participer au développement local car ils ont opéré, en ces temps où l'entreprise individuelle est dominante (et donc les risques très importants), un fort investissement personnel sur place et ils ont donc la volonté de rentabiliser cet investissement qu'ils espèrent pérenne.

\section{B - Des limites à la puissance politique des industriels}

Cette implication des industriels dans la politique locale comporte cependant des limites qui s'affirment avec le temps :

- la première limite est l'opposition croissante des syndicats qui dénoncent un contrôle économique et politique de la population ouvrière, puis de la société entière, notamment après la crise des années 1930 mais surtout avec celle des années 1970. Les jugements négatifs utilisant les termes « seigneur ", « maître », fief » se multiplient alors, dévalorisant un paternalisme autrefois ressenti comme une amélioration sociale certaine ;

- la seconde limite est le déclin de cette implication des industriels dans les affaires politiques locales, notamment après la Première guerre mondiale : les industriels deviennent peu à peu plus des financiers que des entrepreneurs locaux. De plus, peu à peu, des industriels et/ou financiers venus d'autres régions, voire d'autres pays, rachètent des entreprises locales en difficultés et montent des groupes régionaux ou nationaux. Ces groupes, s'ils maintiennent certes le paternalisme, ne se préoccupent plus que de loin de politique locale.

Un des exemples les plus emblématiques de cette évolution est le cas de Marcel Boussac, homme d'affaires (plus qu'industriel) du Centre de la France, ayant racheté de nombreuses usines textiles vosgiennes en difficulté, notamment dans les années 1930 durant la crise. Considéré comme très paternaliste et donc très impliqué économiquement et socialement, il ne l'est pas politiquement, tout du moins au niveau local, dans les communes où il possède une usine. Néanmoins, ses directeurs ou cadres locaux continuent souvent à fournir le personnel politique communal.

Lorsqu'ils accèdent au pouvoir municipal, de nombreux industriels façonnent le territoire dont ils ont la charge politique. L'impact territorial de ces fonctions politiques est alors d'autant plus fort que le pouvoir est exercé à plusieurs échelles et durant plusieurs mandats.

\section{II - Les industriels aménagent le territoire}

\section{A - La création de villes-usines}

19 Les modifications territoriales des communes d'accueil de l'industrie sont d'abord liées à la production, c'est-à-dire au site industriel lui-même et, en particulier, à l'usine. L'industriel choisit le site en fonction d'opportunités foncières certes, mais surtout en fonction des contraintes énergétiques et d'alimentation en eau (proximité d'un cours 
d'eau) et de transport (raccordement possible à une voie ferrée, à un canal...). Il n'existe pas encore à l'époque de documents d'urbanisme et les sites industriels ne sont donc pas regroupés dans des zones prévues à cet effet par les autorités municipales.

Ces usines sont construites selon des modèles architecturaux récurrents (usine en briques à étages, usines aux toits à sheds, etc.) mais n'offrent pas la même monotonie que les hangars aveugles en béton ou à ossature métallique actuels, dont l'usage se répand d'ailleurs largement au delà de l'industrie (Renard-Grandmontagne, 2004). Cette signature paysagère très reconnaissable de l'usine peut encore être appuyée par la volonté de l'industriel de se distinguer de ses concurrents pour des raisons commerciales, mais aussi pour des questions d'affirmation de sa réussite et de sa puissance, à la fois face à ses ouvriers et face aux autorités locales. Ainsi certaines usines présentent-elles une architecture remarquable, utilisant des techniques novatrices et incorporant des éléments de décoration divers peu utiles à la seule efficacité de la production.

Quelque soit leur architecture, les usines de l'époque s'entourent en plus de beaucoup d'autres choses que l'on ne trouve plus aujourd'hui.

Figure 2 : La construction d'une ville-usine à partir de l'exemple de l'usine de wagons De Dietrich à Lunéville (Lorraine) en 1936

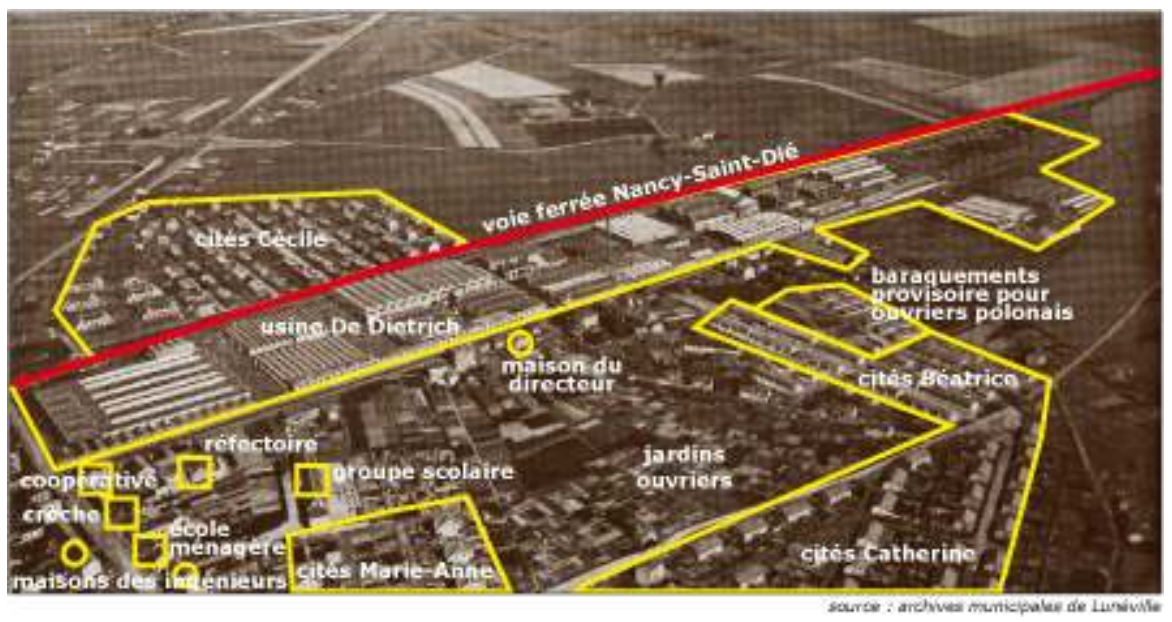

En effet, l'industriel se fait aménageur et est à l'origine de la construction (fig. 2) :

- de logements, essentiellement pour les ouvriers (les fameuses cités ouvrières), mais aussi pour le personnel d'encadrement et pour la direction ;

- d'équipements économiques (magasins, coopératives, économats, fermes...) et sociaux (crèches, écoles, foyers, stade...) ;

- d'infrastructures diverses (voies ferrées de raccordement, port...).

23 Il se constitue ainsi, parfois lentement si l'usine est là depuis longtemps, parfois très rapidement dans le cadre d'implantations programmées ${ }^{2}$, fréquentes à la charnière des $\mathrm{XIX}^{\text {ème }}$ et XX ${ }^{\mathrm{ème}}$ siècles, une ville-usine, système territorial cohérent qui se superpose et s'impose aux organisations spatiales agricoles précédentes.

Cette action des industriels, largement financée par l'usine, est liée d'abord aux carences des communes et plus largement des collectivités locales qui ne peuvent prendre en charge la construction massive de logements pour répondre à l'arrivée brutale de plusieurs centaines d'ouvriers sur un site. L'équipement socio-économique suit : les ouvriers ont besoin d'être approvisionnés, ont besoin d'un encadrement social, 
etc. Certains industriels, influencés par le catholicisme social, développent très largement ces équipements, bien au-delà du strict nécessaire, construisant colonies de vacances, maisons de retraites, stades, etc. Ainsi, c'est finalement l'entreprise qui pallie les carences de l'État et des collectivités locales qui, peu à peu seulement, prennent le relais, avec le développement du socialisme municipal et, plus largement, de l'Étatprovidence.

En retour, ce système permet à l'industriel - et c'est ce qui lui est reproché aujourd'hui avec vigueur - de maîtriser les flux de main d'œuvre (un ouvrier disposant d'un logement en cité sera moins enclin à partir facilement de l'entreprise) et de contrôler les ouvriers. On sait qui participe ou pas aux activités sociales de l'entreprise ; on sait où les ouvriers font leurs courses; on sait qui passe du temps dans son jardin de préférence à aller au café (ou l'inverse)... tout en captant certains flux financiers liés aux achats dans les économats ou aux loyers.

C'est bien un véritable contrôle social de la population qui est mis en place, mais il est difficile de déterminer s'il est lié à une volonté délibérée de l'industriel où s'il découle simplement de la nécessité pragmatique d'encadrement en l'absence d'une politique municipale - voire nationale - en la matière.

\section{B - Une action plus large sur le milieu d'accueil des firmes}

Cette action territoriale des industriels à grande échelle est poursuivie, parfois de manière encore plus spectaculaire même si moins systématique et moins exclusive, à l'échelle départementale. Les industriels vosgiens, par exemple, sont à l'origine de la création, via leur syndicat patronal du textile, d'une école de filature et tissage à Épinal en 1905. Par ailleurs, ces mêmes industriels du textile ont milité pour que le tracé du canal des Vosges ${ }^{3}$ remonte la vallée de la Moselle jusqu'Épinal pour approcher au plus près des usines textiles d'alors et ainsi faciliter leur approvisionnement et l'expédition de leur production. Cela s'est fait aux prix d'un franchissement difficile de l'interfluve entre les bassins de la Moselle et de la Saône, alors que des passages plus faciles et moins coûteux existaient.

L'exercice d'un pouvoir politique d'échelon supérieur (conseiller général, député ou sénateur), allié à leur présence dans de nombreux conseils d'administration de compagnies ferroviaires, permet aux industriels de participer activement aux choix des tracés de ces voies ferrées, capitales pour l'approvisionnement de leurs usines.

Ainsi, Xavier Mougin, patron de la verrerie de Portieux (Vosges) depuis 1867, maire de la commune de 1869 à 1973, conseiller général du canton de Charmes de 1877 à 1895 et enfin député des Vosges de 1889 à 1902 (Grivel, 2006), est aussi actionnaire de la compagnie qui construit la voie ferrée Charmes-Rambervillers. Il parvient à faire passer celle-ci sur son site industriel, auparavant particulièrement difficile d'accès car au fond d'une vallée encaissée au sein d'une vaste forêt.

Ces actions d'aménagement territorial de grande ampleur modifient donc les territoires fonctionnels et contribuent, in fine, à modifier les territoires institutionnels, c'est-à-dire les territoires politiques eux-mêmes. 


\section{III - Les industriels dessinent les territoires politiques}

\section{A - De nouveaux territoires fonctionnels}

31 À la Révolution, les communes françaises sont, pour la grande majorité d'entre elles, nées sur les paroisses de l'Ancien régime elles-mêmes constituées sur les finages lentement construits à partir du Moyen-âge. Ces petits territoires politiques sont donc fondés sur des entités, des systèmes agricoles qui rassemblent des fermes, groupées en un village ou dispersées, et des terres mises en valeur par ces fermes, le tout comprenant une église qui consacre la paroisse et une mairie qui symbolise la commune. Parallèlement, quelques villes, gros centres de commerce des produits agricoles et artisanaux des campagnes voisines, parfois centres administratifs, ont donné naissance à de rares et souvent peu peuplées communes urbaines.

L'arrivée brutale de l'industrie, comme l'urbanisation rapide qui en découle, bouleverse ces systèmes agricoles ou urbains préindustriels, d'abord parce que les usines s'implantent, sans tenir compte des limites communales préexistantes, sur plusieurs communes à la fois, ce qui complique fortement la gestion des communes en question (fig. 3).

Figure 3 : L'inadéquation entre site industriel et limites communales, l'exemple de l'ancienne aciérie ArcelorMittal de Gandrange (Lorraine)

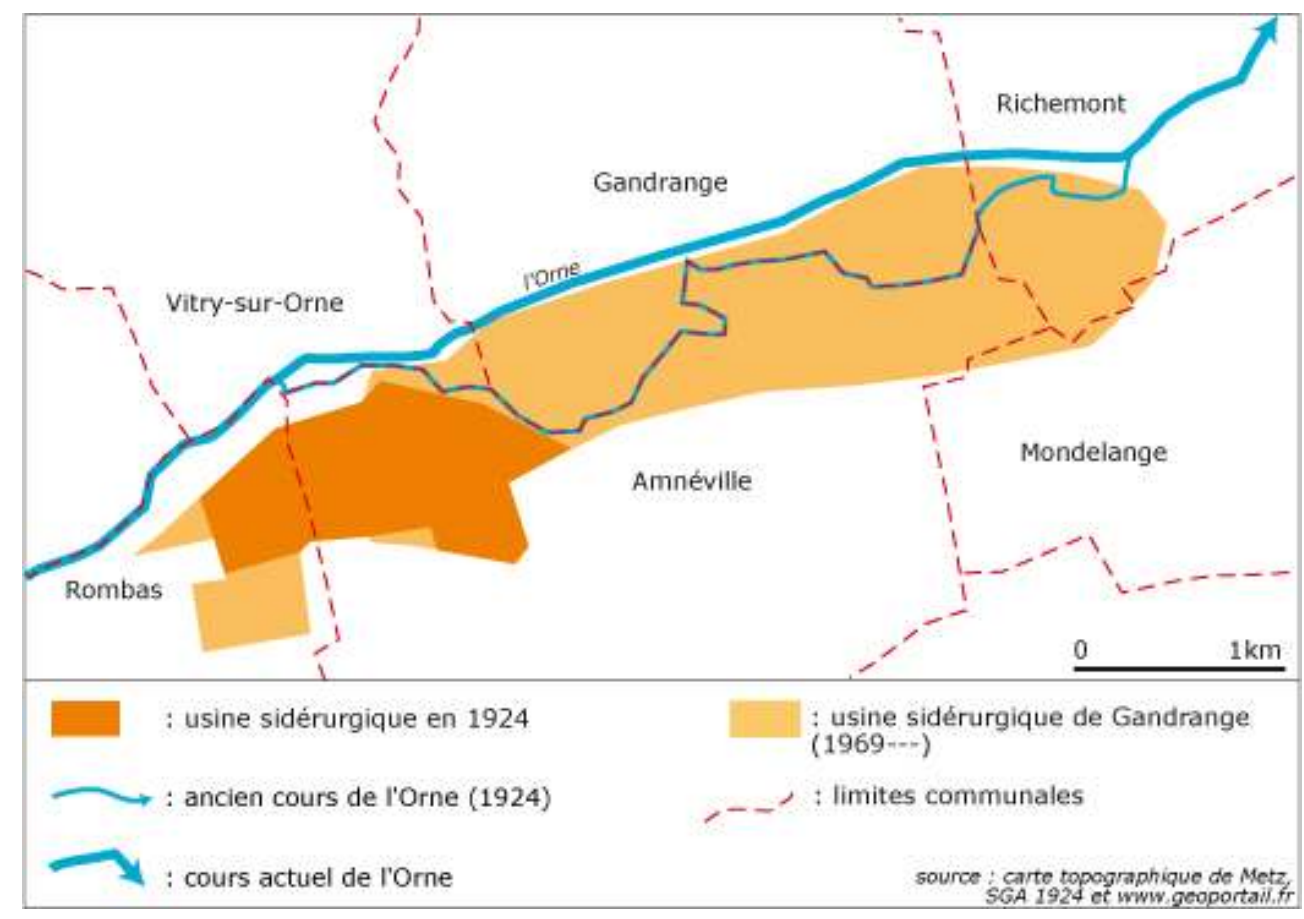

Elles sont en effet construites toujours en fonction des contraintes de production et de logistique plutôt que sur des critères administratifs. La localisation près d'un cours d'eau, à proximité d'une voie ferrée ou d'un gisement prime évidement sur les limites communales. Sur la figure, la grande usine, en partie fermée en 2009 et qui s'étendait sur 6 communes, est récente (1969), mais elle est l'héritière d'anciennes implantations qui remontent à la seconde révolution industrielle et qui étaient déjà transcommunales. 
De plus, les communes sont encore gouvernées, à l'arrivée des usines, par des maires issus du monde agricole et comportent une part plus ou moins forte de population agricole qui n'accepte pas toujours facilement la greffe de population ouvrière. Inversement, les nouveaux habitants au mode de vie ouvrier si différent des ruraux, n'acceptent pas forcément facilement d'être dirigés par des agriculteurs aux préoccupations forts différentes.

Or, pour les entrepreneurs, il est essentiel que l'usine soit sur une seule commune ou sur le moins de communes possible pour en faciliter la gestion. La seule solution, avant la mise en place d'intercommunalités efficaces à la fin du $\mathrm{XX}^{\text {ème }}$ siècle $^{4}$, est la modification des territoires communaux, adaptant les territoires politiques aux territoires fonctionnels, pour éviter une "inadéquation entre le territoire civique légal où intervient la municipalité et le territoire du quotidien » (Dréano, 1996-98).

\section{B - De nouveaux territoires politiques}

36 Ces modifications des territoires communaux sont de deux types (Edelblutte, 2006) : des modifications mineures (échanges, annexions, transferts) ou des modifications majeures (créations).

\section{Des modifications mineures (échanges, annexions, transferts)}

Certaines communes adaptent leurs limites en échangeant ou en annexant des territoires avec ou sur les communes voisines (fig. 4 et fig. 5).

Figure 4 : L'industrialisation provoque une modification mineure de la maille communale : l'échange de 1873 entre Thaon et Girmont

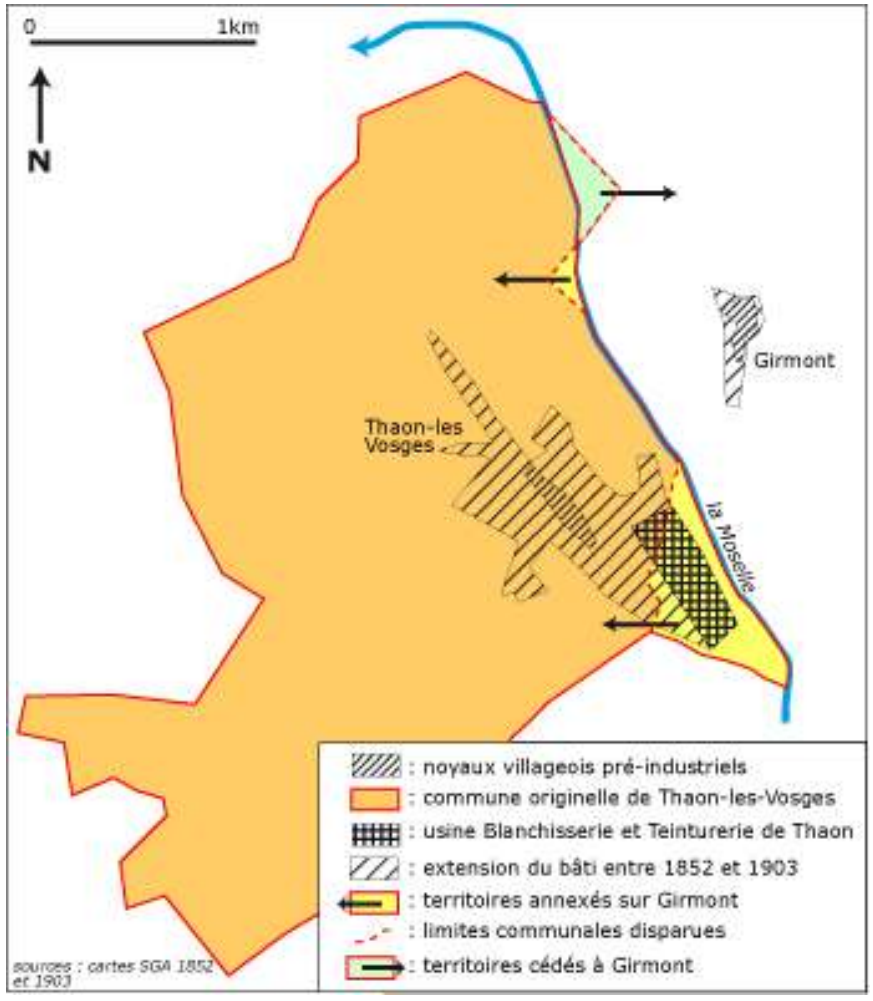


Ainsi, dans les Vosges, à Thaon ${ }^{5}$, l'usine est en partie bâtie en 1872 sur le territoire de la commune voisine de Girmont. 33ha, situés sur la rive gauche de la Moselle, sont concernés. Armand Lederlin, patron de l'usine - et ensuite maire de Thaon à partir de 1884 - demande donc le rattachement des 33 ha de Girmont à Thaon « pour n'avoir affaire qu'à une seule municipalité et n'être assujetti qu'à un seul et même régime de contributions et de juridiction » (Ferry, 1992). Cette annexion est appuyée par la municipalité de Thaon qui trouve alors plus juste, en tant que commune d'accueil de l'usine ayant dû engager de grands frais pour l'installation de cette dernière et des infrastructures lui étant liés, de percevoir la totalité des contributions payées par l'industriel. Pour faire accepter plus facilement cette annexion, la commune de Thaon propose, en échange des 33ha, 8ha thaonnais se trouvant sur la rive droite de la Moselle. L'échange inégal s'effectue dès 1873.

Figure 5 : L'agrandissement du territoire d'une commune industrielle, Le Creusot en 1856

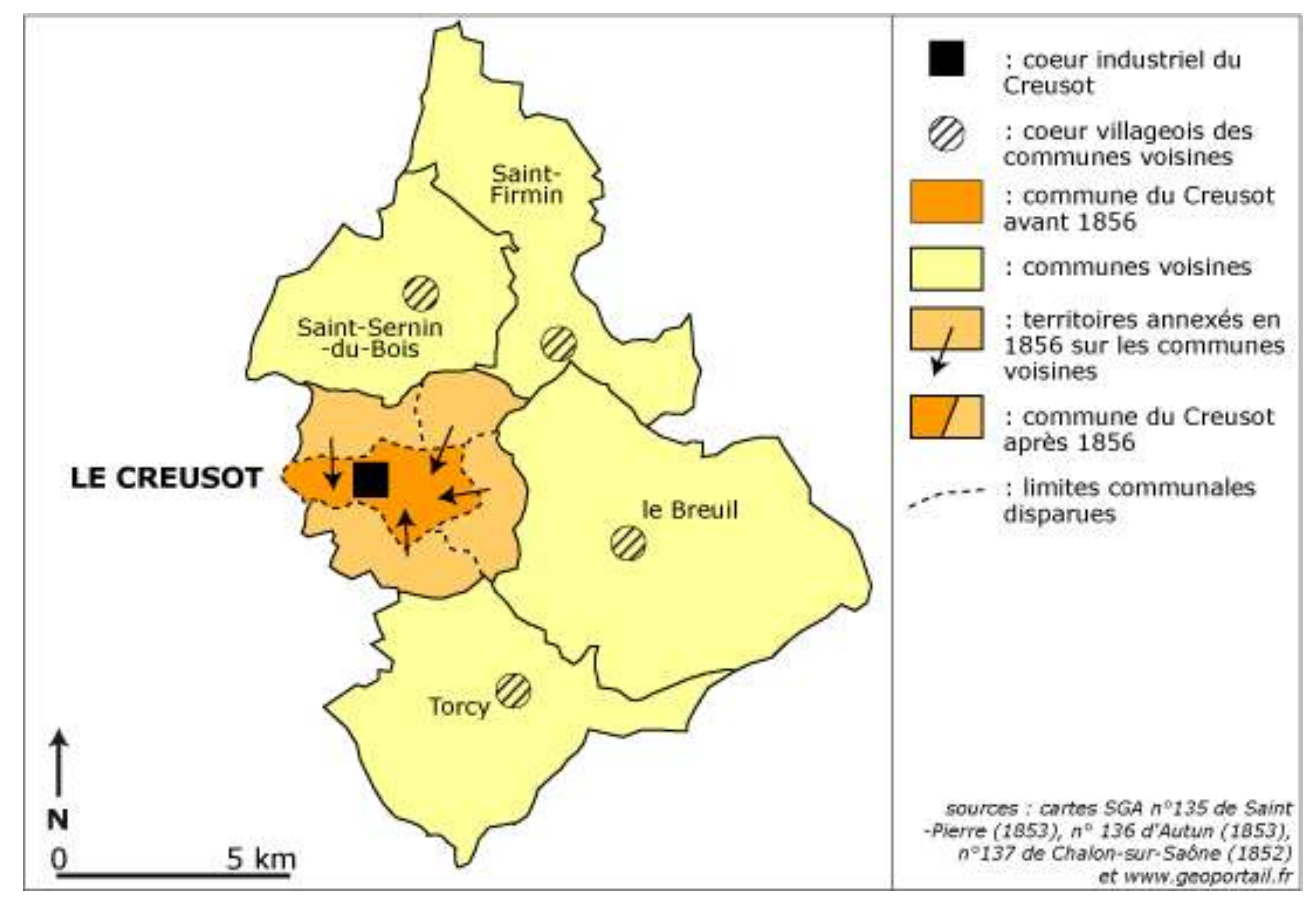

Dans le cas du Creusot (fig. 5), on essaye également de faire coïncider le territoire communal avec un système industriel qui s'étend. L'usine est ancienne, née avant la Révolution (1785). C'est d'ailleurs le berceau de la révolution industrielle en France (Bergeron, 2001). Ainsi, une paroisse (assez petite) avait déjà été crée au XVIII ${ }^{m e}$ siècle autour de l'implantation industrialo-urbaine.

Cette paroisse devient naturellement une commune en 1790. Mais il s'agit d'une petite commune qui ne peut contenir toutes les cités ouvrières et éléments annexes liés à l'usine qui débordent donc rapidement du territoire initial. Ainsi, des parties de communes voisines, où se sont étendus bâtiments industriels, cités, bâtiments économiques et sociaux et infrastructures diverses liés à l'usine, sont annexées au Creusot qui s'agrandit donc en 1856 aux dépens des communes voisines restées plus rurales. 
Enfin, les modifications du territoire politique sont parfois encore plus réduites, comme des changements de chef-lieu, avec le transfert de la mairie d'un village resté agricole vers un écart qui s'est développé avec l'industrie (fig. 7).

\section{Des modifications majeures (créations)}

L'industrialisation provoque aussi la naissance de nouvelles communes. Cela s'effectue de deux façons :

- soit par division simple d'une commune, la partie industrielle se séparant de la partie rurale ou urbaine (fig. 6) ;

- soit par divisions multiples à partir de plusieurs communes voisines rurales (fig. 7).

Figure 6 : Exemples de création de communes par division simple

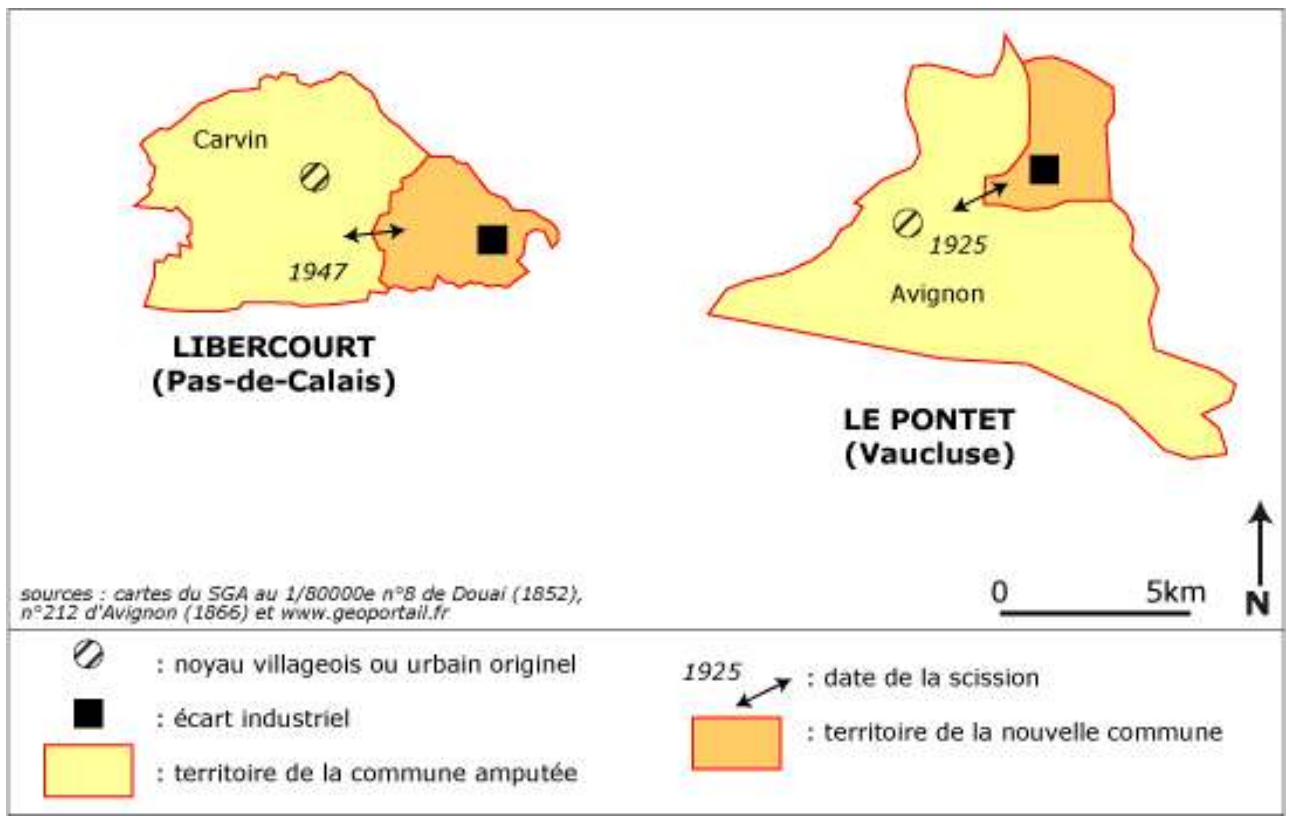


Figure 7 : Exemples de création de communes par divisions multiples

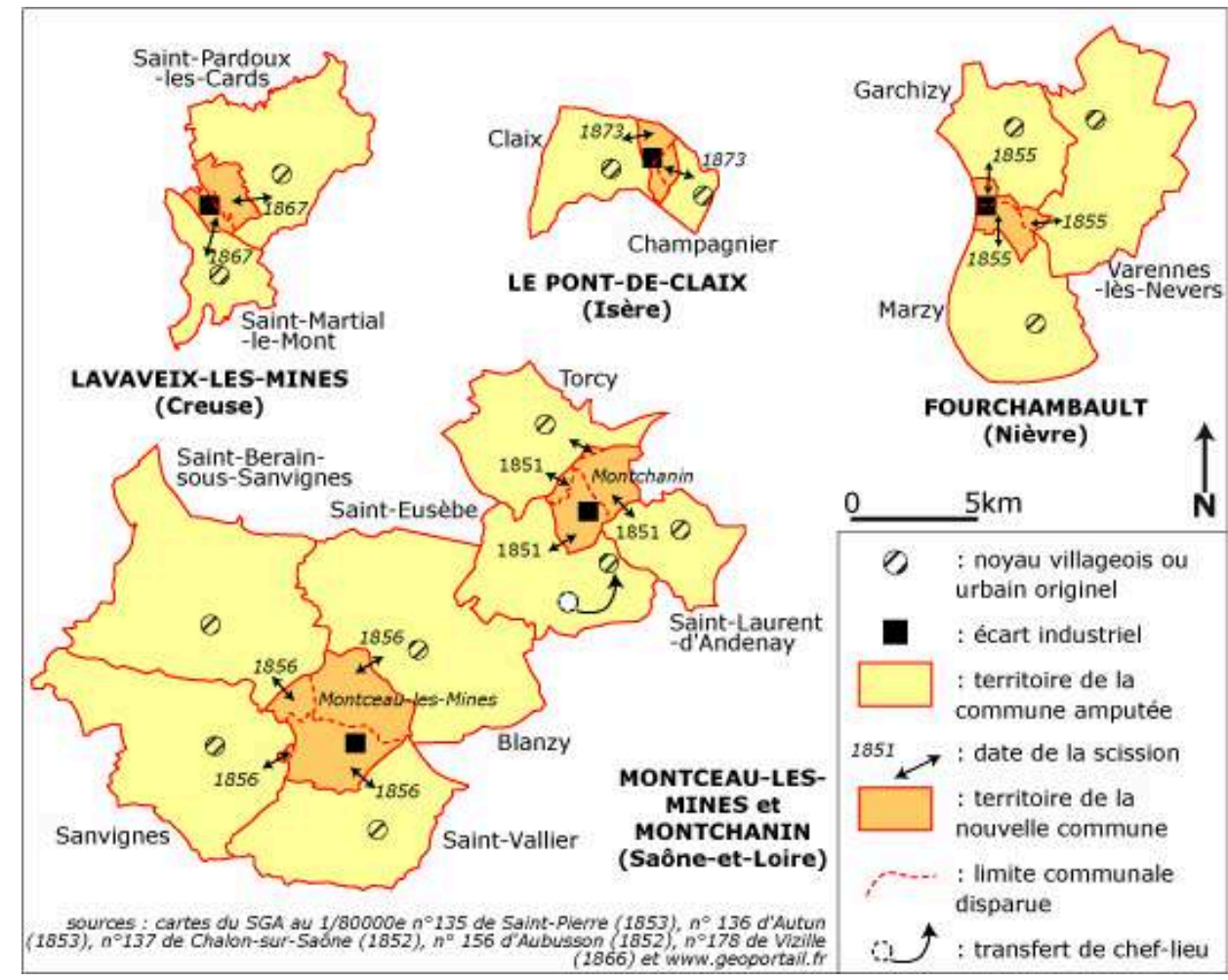

43 Les exemples sont nombreux et, à chaque fois le jeu des industriels est clair et évident dans toutes ces modifications: chacun de ces cas révèle en effet l'importance de la présence d'un "leader indépendantiste ", c'est-à-dire un personnage puissant et charismatique capable de faire plier les autorités et de porter le dossier de l'agrandissement et/ou de la création communale. Ce leader est en général le patron de l'usine, comme A. Lederlin à Thaon, ou lui est lié, comme M. Thomas, au Pontet, «riche héritier oisif d'un ligne industrielle avignonnaise »(Dréano, 1996-98). Il devient la plupart du temps maire de la commune après sa création.

Ces communes sont d'ailleurs si liées au système industriel qui leur a donné naissance, qu'elles se trouvent gravement fragilisées lors de la fermeture de la mine ou de l'usine qui leur avait donné naissance, en général lors de la crise du système fordiste à partir des années 1960-70. Si elles n'ont pas d'activités relais, en particulier lorsqu'elles sont isolées en milieu montagnard ou rural, elles périclitent dangereusement, sont démographiquement dévitalisées et, à plus long terme, pourraient être menacées de disparition (Edelblutte, 2009). Plus près de grandes agglomérations pouvant fournir des emplois, elles maintiennent en général leur population (leur existence n'est donc pas menacée), mais elles deviennent dépendantes de ces agglomérations, à l'instar de banales banlieues-dortoir (tab. 2). 
Tableau 2 : L'évolution de la population dans quelques communes nées de l'industrialisation

\begin{tabular}{|c|c|c|c|c|c|}
\hline $\begin{array}{l}\text { Localisation de } \\
\text { la commune }\end{array}$ & $\begin{array}{c}\text { Commune } \\
\text { (département) }\end{array}$ & $\begin{array}{l}\text { Population lors } \\
\text { de la création } \\
\text { (date du } \\
\text { premier } \\
\text { recensement) }\end{array}$ & $\begin{array}{l}\text { Maximum } \\
\text { démographique } \\
\text { (date) }\end{array}$ & $\begin{array}{c}\text { Population au } \\
\text { dernier } \\
\text { recensement } \\
(2008)\end{array}$ & $\begin{array}{c}\text { Perte de } \\
\text { population par } \\
\text { rapport au } \\
\text { maximum } \\
\text { démographique } \\
(\%)\end{array}$ \\
\hline \multirow{3}{*}{$\begin{array}{l}\text { Proche d'une } \\
\text { agglomération }\end{array}$} & $\begin{array}{c}\text { Fourchambault } \\
\text { (Nièvre) }\end{array}$ & $\begin{array}{c}5380 \\
(1856)\end{array}$ & $\begin{array}{r}6625 \\
(1975) \\
\end{array}$ & 4842 & -27 \\
\hline & $\begin{array}{c}\text { Libercourt } \\
\text { (Pas-de-Calais) }\end{array}$ & $\begin{array}{c}6170 \\
(1946)\end{array}$ & $\begin{array}{l}10726 \\
(1961)\end{array}$ & 8924 & -17 \\
\hline & $\begin{array}{l}\text { le Pont-de-Claix } \\
\text { (Isère) }\end{array}$ & $\begin{array}{r}1032 \\
(1876)\end{array}$ & $\begin{array}{l}13035 \\
(1975)\end{array}$ & 11590 & -11 \\
\hline \multirow{3}{*}{ Isolée } & $\begin{array}{l}\text { Bessèges } \\
\text { (Gard) }\end{array}$ & $\begin{array}{r}7055 \\
(1861) \\
\end{array}$ & $\begin{array}{l}11404 \\
(1881)\end{array}$ & 3282 & -71 \\
\hline & $\begin{array}{l}\text { la Grand-Combe } \\
\text { (Gard) }\end{array}$ & $\begin{array}{l}4011 \\
(1846)\end{array}$ & $\begin{array}{l}13331 \\
(1896)\end{array}$ & 5341 & -60 \\
\hline & $\begin{array}{l}\text { Lavaveix-les- } \\
\text { Mines } \\
\text { (Creuse) }\end{array}$ & $\begin{array}{c}3247 \\
(1872)\end{array}$ & $\begin{array}{c}4108 \\
(1876)\end{array}$ & 846 & -79 \\
\hline
\end{tabular}

Les firmes de la seconde révolution industrielle sont donc bien à l'origine d'importantes et nombreuses modifications des territoires politiques de la grande échelle. Leur action géopolitique constitue une adaptation, à leur profit, du territoire institutionnel au territoire fonctionnel.

\section{Conclusion}

Cette étude montre d'abord que l'influence des firmes sur les territoires politiques n'est pas récente et donc que la longue durée ne peut être négligée sur la thématique liant firmes et géopolitique. D'abord poussé par la nécessité et la simple pression démographique exercée par les ouvriers qu'ils ont recrutés, les industriels ont vite compris l'intérêt qu'ils avaient à s'occuper de politique et notamment de géographie politique et donc de modifier les territoires, de les aménager à leur profit et, plus indirectement et de façon moins évidente, au profit de leurs employés.

Ils ont ainsi agit sur deux plans :

- d'abord en aménageant et équipement concrètement les territoires communaux où ils s'étaient implantés ;

- puis en les modifiant, dans des tentatives d'adapter les territoires institutionnels, politiques, aux territoires fonctionnels, notamment socio-économiques.

Cet interventionnisme aux motivations complexes, mêlant pragmatisme et volonté de contrôle politico-social, provoque une fertilisation croisée entre l'entreprise, qui profite de ces aménagements et du contrôle social, la collectivité locale, qui se voit financer certains équipements, et la population, qui les utilise. Elle est à la base de la naissance de villes-usines.

Néanmoins, elle repose sur un équilibre fragile qui est vite rompu :

- lors de crises économiques qui affectent, voire font disparaître, l'entreprise ;

- lors de bouleversements politiques qui portent au pouvoir, dans un contexte d'intervention grandissante de l'État dans la sphère sociale, des municipalités moins favorables à l'industriel local.

50 Aujourd'hui cependant, les entreprises participent toujours à la vie des communes, mais par des interventions moins visibles et plus indirectes (financement, aides versées aux communes...). Certains dirigeants d'entreprises sont encore maires et interviennent dans l'aménagement de leur commune, mais ils ne le font plus dans 
l'intérêt de leur entreprise (tout au moins ne sont-ils plus censés le faire...) et, surtout, ils ne modifient plus les territoires politiques de la grande échelle, qui sont d'ailleurs de plus en plus contournés par l'intercommunalité. Globalement, les entreprises sont donc bien moins impliquées qu'autrefois dans la gestion des communes. Cet éloignement entreprise/commune d'accueil est souvent déploré à un moment où les décisions de localisation/délocalisation sont prises depuis des sièges sociaux éloignés des communes où sont implantées les usines, coupant les employés de la direction et exonérant ainsi plus facilement celle-ci de toute responsabilité sociale.

\section{BIBLIOGRAPHIE}

Bergeron L. (2001), Le Creusot, une ville industrielle, un patrimoine glorieux, Paris, BelinHersher, $192 \mathrm{p}$.

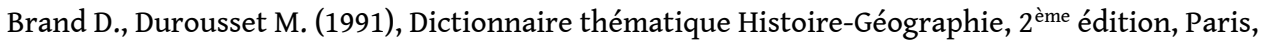
Sirey, $507 \mathrm{p}$.

Donze J. (2001), L'Argentière-La-Bessée, de la reconversion au redéveloppement, Hommes et Terres du Nord, $\mathrm{n}^{\circ} 1-2001$, p. 39-45.

Di Meo G. (1993), Les territoires de la localité, l'Espace Géographique, Tome XXII - nº, p. 306-318.

Dreano J.-M. (1996-98), Affirmation d'une identité locale de banlieue : l'érection en commune du quartier de Montfavet (Avignon), Bulletin de la Société de Géographie de Marseille, Tome XCIII $n^{\circ} 21$, p. 69-82.

Dreano J.-M. (1997), Les discours sur la commune française : les voies d'un consensus. Le cas du Midi méditerranéen de 1884 à nos jours, Histoire et Mesure, 3-4/1997, Tome XII, p. 337-360.

Dupaquier J., Felkay N., Guerout J., Jacquard J., Lachiver M., Lemee R., Rollet C., Souriac A. (1974), Paroisses et communes de France - Région parisienne, Paris, CNRS éditions, $922 \mathrm{p}$.

Durupt P. (1990), Hommes et femmes du textile dans les Hautes-Vosges, Remiremont, Société d'Histoire Locale de Remiremont et de sa Région, $192 \mathrm{p}$.

Edelblutte S. (2009), Que sont devenues les villes-usines ? Réflexion à partir du cas lorrain. In : VALLAT Colette - dir, DUFAUX Frédéric, LEHMAN-FRISCH Sonia - coord., Pérennité urbaine, ou la ville par-delà ses métamorphoses, vol. 3 : Essences, Paris, L'Harmattan, p. 137-153.

Edelblutte S. (2006), Géohistoire de l'adaptation de la maille communale à l'organisation spatiale. L'exemple des villes-usines. In : BLETON-RUGET Annie, COMMERÇON Nicole, GONOD Philippe dir., Territoires institutionnels, territoires fonctionnels, Institut de Recherche du Val de SaôneMâconnais, p. 239-252.

El Gammal J. - dir. (2006), Dictionnaire des parlementaires lorrains de la Troisième République, Metz, Éditions Serpenoise, 422p.

Ferry C. (1992), La Blanchisserie Teinturerie de Thaon - 1872-1914, Presses Universitaires de Nancy, Nancy, 304 p. 
Grivel G. (2006), Mougin (Xavier) - 1837-1912. In : EL GAMMAL J. - dir. (2006), Dictionnaire des parlementaires lorrains de la Troisième République, Metz, Éditions Serpenoise, p. 391-392.

Houssel J.-P. (1992), L'industrialisation d'une région rurale : l'exemple du canton de SaintLaurent-de-Chamousset (Rhône), Revue de Géographie de Lyon, volume, n³, p. 219-229.

Maisonniac N. (2000), Un pôle industriel au bout du monde : les Boutières (Ardèche), Revue de l'Économie Méridionale, vol. 48, nº189-90, p. 63-76.

Mort d'Yves Rocher : La Gacilly, fief breton du géant de la beauté, www.lepoint.fr, publié le $26 / 12 / 2009$

Poull G. (1982), L'industrie textile vosgienne (1765-1981), Rupt-sur-Moselle, Édité par l'auteur, $474 \mathrm{p}$.

Renard-Grandmontagne C. (2004), Les zones d'activité : espaces pionniers à la périphérie des agglomérations. Le cas lorrain, Thèse de doctorat en géographie, Université Nancy2, Centre d'Études et de Recherches sur les Paysages, 458 p.

Vandermotten Ch., Marissal P. (2004), La production des espaces économiques (Tomes 1 \& 2), $2^{\text {ème }}$ édition, Bruxelles, Université de Bruxelles, 295 p. \& 468 p.

www.francegenweb.org/mairesgenweb, site généalogique ayant pour objectif (encre largement incomplet en 2010), de recenser les maires de chaque commune.

\section{NOTES}

1. Le terme " industriel » a été préféré à celui de " patron », utilisé plus souvent à l'époque, mais aujourd'hui politiquement connoté. Il traduisait cependant bien la réalité d'une époque où l'entreprise restait encore très largement confondue avec son propriétaire et directeur.

2. Les Bataville, installées dans le monde entier par l'entreprise de chaussures Bata, alors tchécoslovaque, ont été construites de toutes pièces en milieu rural et sont des exemples emblématiques de ces ville-usines programmées.

3. Le canal des Vosges est l'ancien canal de l'Est branche Sud, construit après 1870 pour rétablir les liaisons fluviales de l'Est de la France suite à l'annexion de l'Alsace-Moselle.

4. Il n'existe pas encore, au XIXème siècle, de coopération intercommunale institutionnalisée. Elle n'était en effet pas nécessaire puisque, jusqu'à l'irruption de l'industrie, les communes, territoires institutionnels, correspondaient aux finages, territoires fonctionnels.

5. Cette commune deviendra ensuite "Thaon-les-Vosges", à la demande d'Armand Lederlin et pour éviter la confusion avec un Thaon normand.

\section{RÉSUMÉS}

Ce travail est conçu comme un regard rétrospectif sur les liens entre les firmes et les territoires politiques de la grande échelle, c'est-à-dire les communes. Il veut montrer les différentes formes d'action politique, en particulier d'action géopolitique par la modification des territoires, d'acteurs en premier lieu économiques. 
Dès la constitution des grandes entreprises industrielles au XIX ${ }^{\text {ème }}$ siècle, leurs patrons sont en effet intervenus dans la vie politique locale, par nécessité, car ni les autorités locales ni les autorités nationales n'avaient l'envergure pour répondre aux besoins de villes-usines champignons, mais aussi par conviction sociale et calcul politique. Ils sont ainsi devenus maires et/ou conseillers généraux.

Cette mainmise sur le pouvoir local a permis à beaucoup d'industriels de développer une politique paternaliste très aboutie, en réalisant des véritables actions d'aménagement du territoire au sein de leur commune : construction de cités ouvrières, des bâtiments de services, de commerces, d'infrastructures diverses, etc.

Enfin, concrétisant ce lien entre firmes et politique, les territoires communaux eux-mêmes ont été transformés par la volonté patronale au temps du paternalisme: agrandissements du territoire, annexions de territoires voisins et créations de communes sont ainsi fréquents autour des villes-usines.

This work is about past links between firms and political territories of the large scale, i.e. French communes. In this context, it aims to show the different forms of geopolitical actions driven by economical stakeholders.

Since the birth of large companies during $19^{\text {th }}$ century, their owners/managers interfered in local politics, by necessity, first because local and national authorities hadn't the stature to meet the needs of new industrial boomtowns, but also by social conviction and political calculation. So they became mayors and/or other local politicians.

This local power drove these owners/managers to develop paternalism, with the building of many workers' houses or many services, shops and diverse elements for workers.

At last, on the top of these links between firms and political territories, the territories of the communes were themselves modified by the local industrial owners/mayors. Extension of territories, annexation of neighboring territories and creation of new communes can be observed around industrial boomtowns of the $19^{\text {th }}$ century.

Zusammenfassung: Diese Arbeit wird konzipiert als eine rückblickende Betrachtung über die Verbindungen zwischen den Firmen und den politischen lokalen Territorien, d. h. den Gemeinden. Es handelt sich darum, die unterschiedlichen Formen politischer Aktionen zu zeigen, insbesondere der geopolitischen Aktionen durch die Änderung der Gemeindegebiete.

Sobald industrielle Großunternehmen im $19 \mathrm{Jh}$. entstanden, haben ihre Besitzer in der lokalen Politik interveniert. Es war für sie nötig, weil weder die lokale noch die nationale Macht die Möglichkeit hatten, den Bedürfnissen der stark wachsenden Industriestädte zu befriedigen. Aber diese Intervention war auch durch soziale Überzeugung und politische Kalkül motiviert. So wurden sie Bürgermeister oder Vertreter im Departement.

Diese Einflußnahme über die lokale Macht hat vielen Industriebesitzern erlaubt, eine sehr erfolgreiche paternalistische Politik zu entwickeln, indem sie Raumordnungsmaßnahmen auf dem Gebiet ihrer Gemeinde durchgeführt haben: Bau von Arbeitersiedlungen, Handelsgebäuden, unterschiedlichen Einrichtungen, usw.

Als Konkretisierung dieser Verbindung zwischen Firmen und Politik wurde in der paternalistischen Zeit das Gebiet der Gemeinden durch die Wille der Industriebesitzer geändert: Erweiterung des Gemeindegebietes, Zusammenschlu $\beta$ der Nachbargemeinden und Schaffung neuere Gemeinden erfolgen häufig um die Industriestädte. 
INDEX

Keywords : communes, company-town, France, paternalism, second industrial revolution Schlüsselwörter : Fabrikstadt, Frankreich, Gemeinden, Paternalismus, zweite industrielle Revolution

Mots-clés : communes, France, paternalisme, seconde révolution industrielle, ville-usine

\section{AUTEUR}

\section{SIMON EDELBLUTTE}

Université Nancy2, CERPA - Centre d'Études et de Recherches sur les Paysages - 23, boulevard Albert I ${ }^{\text {er }}, 54000$ NANCY, simon.edelblutte@univ-nancy2.fr 